\title{
Comparative Study of Pre and Post Monsoon Water Quality in and Around Gandhidham, Kachchh, Gujarat, India, for the Year 2015
}

\author{
Madhu D. Nathani ${ }^{1}$, Dr. Mrugesh M Trivedi ${ }^{2}$ \\ ${ }^{1,2}$ Department of Earth and Environment, KSKV Kachchh University, Bhuj, Gujarat, India
}

\begin{abstract}
The major objective of the present report is to do comparative study of the physiochemical parameters including heavy metal contamination in surface, ground and potable water in industrial, residential and commercial zone in and around Gandhidham, Kachchh. To collect the information on the level of contamination 40 water samples were collected from various zones of Kachchh in and around Gandhidham Taluka, Gujarat during $23^{\text {rd }}$ March and20th April 2015 i.e. Pre Monsoon Season. and $22^{\text {nd }}$ October to $14^{\text {th }}$ November 2015 ie Post Monsoon. Parameters like pH, electrical conductivity, TDS, salinity, total dissolved solids, total hardness, calcium and magnesium hardness, fluoride, potassium, sulphate, nitrate, silicate chloride and metals like Iron, Cadmium, Cobalt, Zinc, Manganese, Nickel and Copper were analysed.
\end{abstract}

Keyword: Water quality, ground water, physico-chemical, Gandhidham and Anjartaluka.

\section{Introduction}

Contamination of aquifers is an increasing problem in several parts of India. This along with scarcity of groundwater resources due to increase in water demand and also by reduction in recharge of groundwater from changing land use, combines to further compound the problem. High Salinity, Fluoride, Nitrate and pollution from industrial effluents have caused contamination of aquifers which beset with numerous quality problems, some of which are increasing in intensity over the years. Largest producer of Salt, Saline desert and gulf regions add sea water intrusion into aquifers is a common problem. Excessive Fluoride is another problem in groundwater. Saline water being denser occupies the lower layer than fresh water. Under equilibrium conditions between fresh inland water and sea water, a depletion of water table can result in the depth of saline water below sea level reduce by forty times as compared with drop in water tables above sea-level. Intensification of groundwater use combined with decreased recharge of fresh groundwater into inland aquifers and high evaporation rate has electrified the problem.

Kachchh, after an earth quake of January, 2001 has various pockets of high industrial activity where large amounts of effluents are released, sometimes directly into wells Toxic substances releases from industries need to be treated according to norms laid by the Pollution Control Board. But, many industries release their effluents without proper treatment causing harmful heavy metals and chemicals to be released into the environment. Lead is detected beyond desired limits in various belts of Kachchh.
Several toxic metals which are important to the environment and human health have been detected in aquatic media. These toxic metals include the non-essential metals and are of no importance to humans (Borgmann and Norwood, 2002). These toxic metals have been found to have accumulated mainly in the kidney and liver and high concentrations have also been found to lead to chronic kidney dysfunction. Steoppler (1997) has shown that when lead is ingested by man, it enters the blood stream and soon it begins to appear in the liver and kidney but ultimately over $90 \%$ of $\mathrm{Pb}$ absorbed end up in bones where it replaces calcium.heavy metal toxicity can cause chronic degenerative diseases the symptoms being mental disorders, pain in muscle and joints, gastro intestinal disorders, vision problems, chronic fatigue, and susceptibility to fungal infections. Sometimes the symptoms are vague and difficult to diagnose at early stage. Genotoxicity and cancers can also occur. Some elements like $\mathrm{Fe}, \mathrm{Zn}, \mathrm{Cu}, \mathrm{Co}, \mathrm{Mn}, \mathrm{Ni}$, are needed in small quantities for human metabolism as they work as coenzymes, but may be toxic at higher levels. Others like lead, mercury, cadmium, and arsenic etc. have no beneficial role and are positively toxic. Small amounts of fluoride help to prevent dental caries, but excess is harmful.

\section{Work Area}

Kutch District forms part of the SAURASHTRA Region of Gujarat State having an area of $45652 \mathrm{Sq}$. Km. the Largest in India. It is situated in the South western corner of the Gujarat between $22.44^{\circ}$ and $24.41^{\circ}$ North Latitude and $78.89^{\circ}$ and $71.45^{\circ}$ East Longitud 
International Journal of Science and Research (IJSR)

ISSN (Online): 2319-7064

Index Copernicus Value (2013): 6.14 | Impact Factor (2015): 6.391

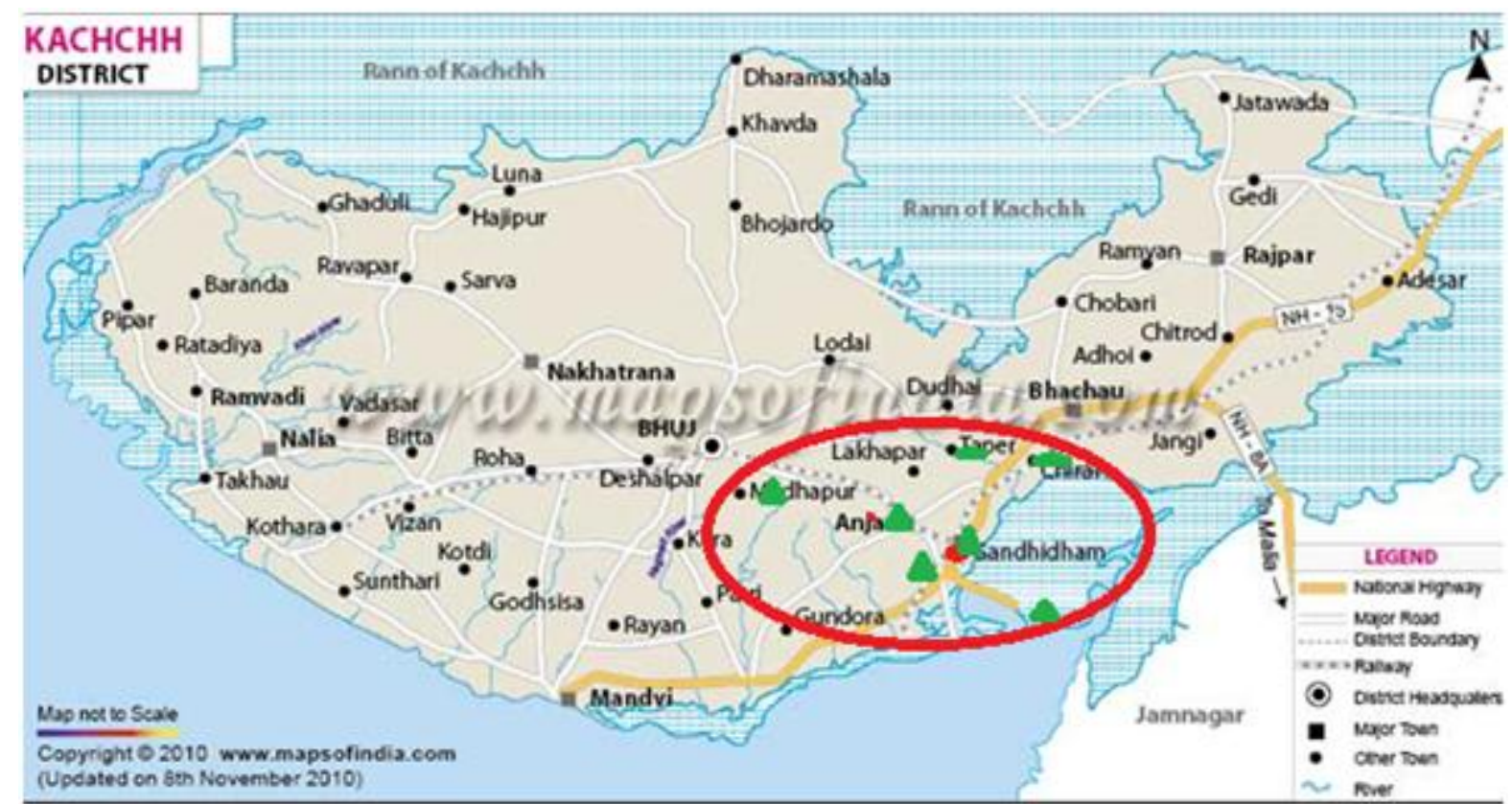

Gujarat

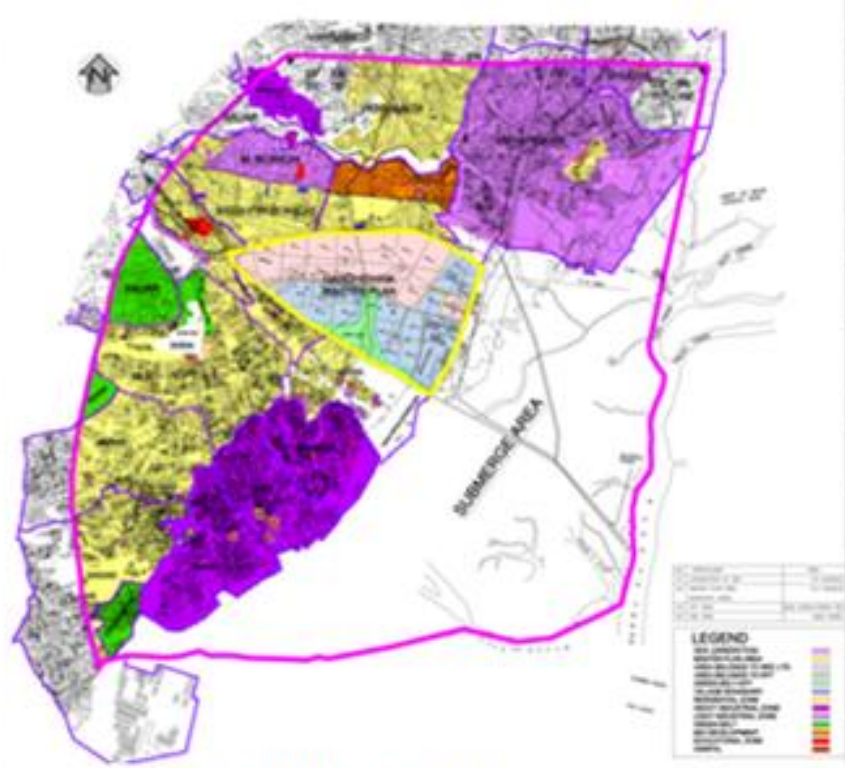

Gandhidham Taluka

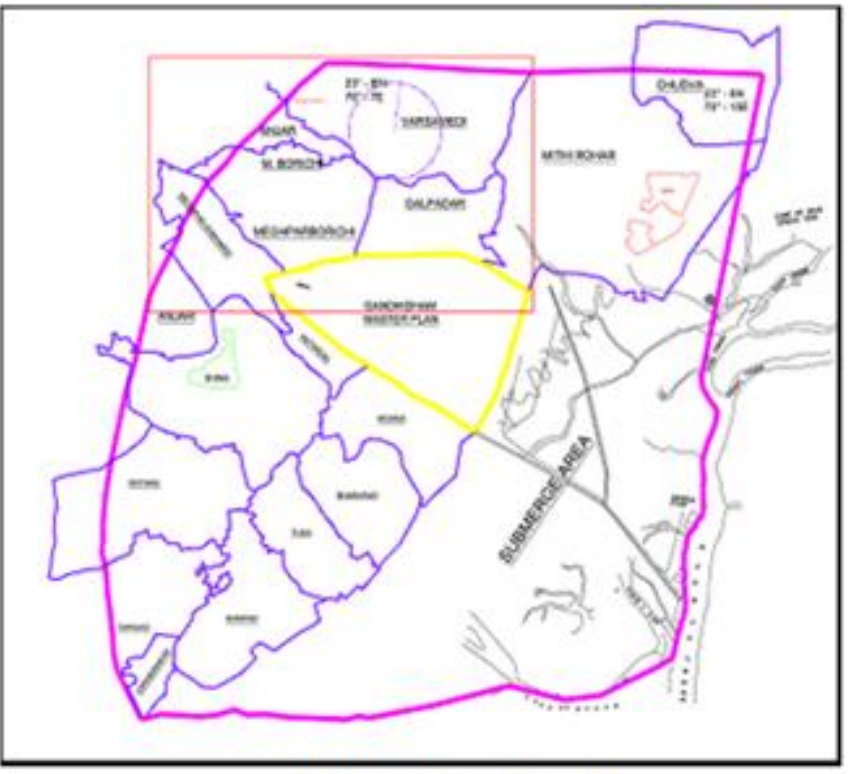

Villages in work area

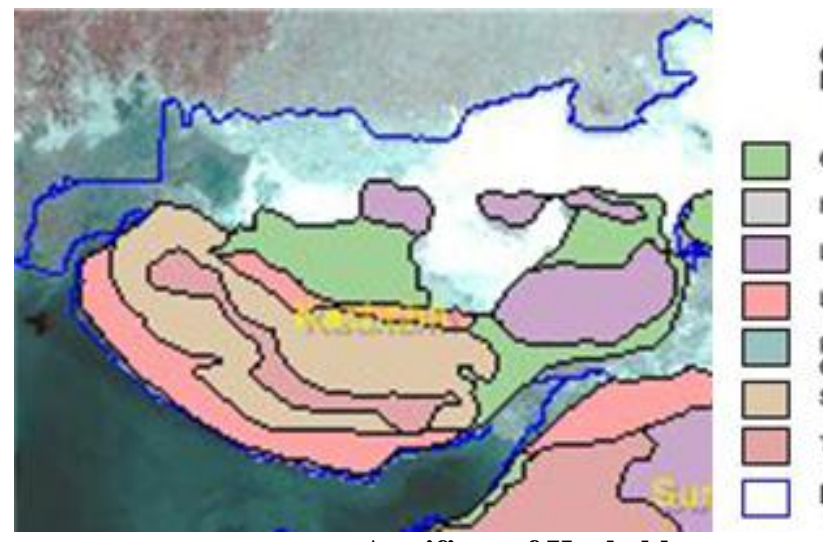

Aquifiers of Kachchh
Ground Water Resources

Coastal Alluvium

Highlevel Alluvium

Limestone and Cloys

Lowlevel Alluvium

Procambrian

Crystalines

Sandstones Laterits

Trappean Basaits

DistrictOld

Volume 5 Issue 6, June 2016 www.ijsr.net

Licensed Under Creative Commons Attribution CC BY 


\section{International Journal of Science and Research (IJSR) \\ ISSN (Online): 2319-7064}

Index Copernicus Value (2013): 6.14 | Impact Factor (2015): 6.391

For this the study area is divided in different zones

\begin{tabular}{|c|c|c|c|}
\hline Sr. No. & Sample site & Type & $\begin{array}{c}\text { General characteristic of } \\
\text { Sample site }\end{array}$ \\
\hline 1 & Anjar & Rural & Residential, Agricultural \\
\hline 2 & Shinai & Rural & Residential, , Agricultural \\
\hline 3 & Sangad & Rural & Residential \\
\hline 4 & Devadia & Rural & Green Belt \\
\hline 5 & MeghparKumbharadi & Rural & Residential, Industrial \\
\hline 6 & MeghparBorichi & Rural & Residential, Industrial \\
\hline 7 & Kandla & Urban & Residential, Industrial, Port \\
\hline 8 & Varsamedi & Rural & Residential, Industrial \\
\hline 10 & Galpadar & Rural & Residential, Industrial \\
\hline 11 & Mithirohar & Rural & Industrial, Residential \\
\hline 12 & Kidana & Rural & Residential \\
\hline 13 & Bharapar & Rural & Industrial \\
\hline 14 & Tuna & Rural & Residential, Industrial \\
\hline 15 & Rampar & Rural & Residential, Industrial \\
\hline 16 & Gandhidham / Adipur & Urban & Residential \\
\hline 17 & Mathak & Rural & Residential, Agricultural \\
\hline 18 & Antarjal & Rural & Residential \\
\hline
\end{tabular}

Analysis Methods Population in last 20 Years

\section{Analysis Methods}

\begin{tabular}{|c|c|c|}
\hline Parameter & Method & Instrument/Apparatus \\
\hline $\mathrm{pH}$ & Potentiometry & \multirow{2}{*}{$\begin{array}{l}\text { Digital pH/EC meter- } \\
\text { Model- LabIndia }\end{array}$} \\
\hline $\mathrm{EC}$ & Conductometry & \\
\hline TDS & Conductometry & Digital TDS Meter \\
\hline Nitrate & Brucine method & \multirow{5}{*}{$\begin{array}{c}\text { UV-VIS } \\
\text { Spectrophotometer }\end{array}$} \\
\hline Phosphate & Ascorbic acid method & \\
\hline Sulphate & Turbidimetry & \\
\hline Fluoride & SPADNS Method & \\
\hline Silicate & Molybdosilicate method & \\
\hline Sodium & & \multirow{2}{*}{ AAS } \\
\hline Potassium & & \\
\hline Calcium & \multirow[t]{4}{*}{ EDTA Titration Method } & ------- \\
\hline Magnesium & & ------- \\
\hline Total Hardness & & \\
\hline Alkalinity & & \\
\hline Chloride & (Titrimetric) & ------- \\
\hline $\begin{array}{l}\text { Heavy metals } \\
(\mathrm{Pb}, \mathrm{Cu}, \mathrm{Cd}, \mathrm{Ni} \text {, } \\
\mathrm{Fe}, \mathrm{Mn}, \mathrm{Ni} \text { and } \\
\mathrm{Co} \text { etc. })\end{array}$ & $\begin{array}{c}\text { Solvent extraction cum } \\
\text { pre-concentration method }\end{array}$ & $\begin{array}{l}\text { Atomic Absorption } \\
\text { Spectrometer }\end{array}$ \\
\hline
\end{tabular}

Population in last 20 years

\begin{tabular}{|c|c|c|c|}
\hline name & 1991 & 2001 & 2011 \\
\hline Anjar & 51,209 & 68,343 & 87,183 \\
\hline Antarjal & & 6,036 & 11,256 \\
\hline Galpadar & & & 13,155 \\
\hline Gandhidham & $1,04,585$ & $1,51,693$ & $2,47,992$ \\
\hline Kandla & 19,787 & 14,695 & 15,782 \\
\hline
\end{tabular}

\section{Results and Discussion}

Comparative study of Pre and Post Monsoon Water Quality

Ground Water(19 Samples)

\begin{tabular}{|c|c|c|c|c|c|c|c|c|}
\hline Sr. No. & $\begin{array}{l}\text { Parameters( all values } \\
\text { one in } m g / l \text { except } p H)\end{array}$ & $\begin{array}{c}\text { Range Pre- } \\
\text { Mon }\end{array}$ & $\begin{array}{c}\text { Range Post- } \\
\text { Mon }\end{array}$ & Std BIS & $\begin{array}{c}\text { \% samples } \\
\text { exceeding } \\
\text { desired } \\
\text { limit-pre } \\
\text { monsoon }\end{array}$ & $\begin{array}{l}\text { \% samples } \\
\text { exceeding } \\
\text { desired } \\
\text { limit-post } \\
\text { monsoon }\end{array}$ & $\begin{array}{c}\text { Range of } \\
\text { Increase } \\
\text { Concentration } \\
\text { after monsoon } \\
(\%)\end{array}$ & $\begin{array}{c}\text { sample } \\
\text { showing } \\
\text { increase after } \\
\text { Monsoon (\%) }\end{array}$ \\
\hline 1 & $\mathrm{pH}$ & $6.75-8.6$ & $6.79-8.96$ & $6.5-8.5$ & & & & \\
\hline 2 & Total Dissolved salt & $1012-5091$ & $803-4035$ & 500 & 100 & 100 & $0.3-47.7$ & 26 \\
\hline 3 & Chloride as $\mathrm{Cl}^{-}$ & 6- 1506 & $105-1327$ & 250 & 89 & 94 & $3.2-1650$ & 63 \\
\hline 4 & Total Alkalinity & $230-1012$ & $115-728$ & 200 & 100 & 94 & $1.5-56.7$ & 47 \\
\hline 5 & Total hardness as $\mathrm{CaCo} 3$ & $69-1199$ & $52-1238$ & 300 & 78 & 84 & $2.3-180$ & 47 \\
\hline 6 & Calcium as $\mathrm{Ca}^{2+}$ & $19-444$ & $36-700$ & 75 & 94 & 89 & $0.2-636$ & 57 \\
\hline 7 & Magnesium as $\mathrm{Mg}^{2+}$ & $50-755$ & $16-798$ & 30 & 100 & 97 & $0.5-36$ & 31 \\
\hline 8 & Silicates as $\mathrm{SiO}_{2}$ & $12.12-74.9$ & $1.88-51.4$ & 17 & & & $2.3-94$ & 36 \\
\hline 9 & Sulphates as $\mathrm{So}_{4}{ }^{2-}$ & $0-423$ & $0-241$ & 200 & 5 & 15 & $29.5-203$ & 31 \\
\hline 10 & Nitrates as $\mathrm{NO}_{3}{ }^{-}$ & $0.9-72.7$ & $1.4-89$ & 45 & 5 & 15 & 2- 1846 & 57 \\
\hline 11 & Sodium & $184-1070$ & $72.7-1036$ & 200 & 100 & 94 & 4-108 & 31 \\
\hline 12 & Potassium & $0.46-18$ & $0-64$ & 1 & 5 & 100 & $21-1107$ & 68 \\
\hline 13 & Fluoride as $\mathrm{F}^{-}$ & $0.34-3.47$ & $0.29-4.12$ & 1 & 26 & 57 & $1.6-291$ & 73 \\
\hline
\end{tabular}

Volume 5 Issue 6, June 2016 www.ijsr.net

Licensed Under Creative Commons Attribution CC BY 


\section{International Journal of Science and Research (IJSR) \\ ISSN (Online): 2319-7064}

Index Copernicus Value (2013): 6.14 | Impact Factor (2015): 6.391

\begin{tabular}{|c|c|c|c|c|c|c|c|c|}
\hline Sr.No. & $\begin{array}{c}\text { Parameters( } \\
\text { all values one } \\
\text { in mg/l })\end{array}$ & Range Pre- Mon Metals & $\begin{array}{c}\text { Range Post- } \\
\text { Mon }\end{array}$ & Std BIS & $\begin{array}{c}\text { \% samples } \\
\text { exceeding } \\
\text { desired limit- } \\
\text { pre monsoon }\end{array}$ & $\begin{array}{c}\% \text { samples } \\
\text { exceeding } \\
\text { desired limit- } \\
\text { post } \\
\text { monsoon }\end{array}$ & $\begin{array}{c}\text { Range of } \\
\text { Increase } \\
\text { Concentration } \\
\text { after monsoon } \\
(\%)\end{array}$ & $\begin{array}{c}\text { sample } \\
\text { showing } \\
\text { increase after } \\
\text { Monsoon } \\
(\%)\end{array}$ \\
\hline 1 & Iron & $0.09-5.63$ & $0.04-1.03$ & 0.3 & 36 & 21 & $40-999$ & 36 \\
\hline 2 & Nickel & $0.004-0.216$ & $0-0.213$ & 0.02 & 21 & 84 & $64-3848$ & 78 \\
\hline 3 & Manganese & $0.012-1.39$ & $0.003-0.103$ & 0.1 & 36 & 10 & $149-330$ & 10.5 \\
\hline 4 & Cadmium & $0.001-0.018$ & $0.004-0.008$ & 0.01 & 26 & 0 & $16-490$ & 36 \\
\hline 5 & Lead & $0-0.143$ & $0.002-0.06$ & 0.01 & 52 & 94 & $45-1343$ & 52 \\
\hline 6 & Copper & $0.004-0.18$ & $0.001-0.011$ & 0.05 & 31 & 0 & $12-206$ & 10 \\
\hline 7 & Zinc & $0.02-0.26$ & $0-0.225$ & 5 & 0 & 0 & $15-866$ & 36 \\
\hline
\end{tabular}

Potable Water (14 Samples)

\begin{tabular}{|c|c|c|c|c|c|c|c|c|}
\hline $\begin{array}{c}\text { Sr. } \\
\text { No. }\end{array}$ & $\begin{array}{c}\text { Parameters( all values } \\
\text { one in } \mathrm{mg} / \mathrm{l})\end{array}$ & $\begin{array}{c}\text { Range Pre- } \\
\text { Mon }\end{array}$ & $\begin{array}{c}\text { Range Post- } \\
\text { Mon }\end{array}$ & $\begin{array}{c}\text { Std } \\
\text { BIS }\end{array}$ & $\begin{array}{c}\text { \% samples } \\
\text { exceeding } \\
\text { desired limit- } \\
\text { pre monsoon }\end{array}$ & $\begin{array}{c}\text { \% samples } \\
\text { exceeding } \\
\text { desired limit- } \\
\text { post monsoon }\end{array}$ & $\begin{array}{c}\text { Range of Increase } \\
\text { Concentration after } \\
\text { monsoon }(\%)\end{array}$ & $\begin{array}{c}\text { sample } \\
\text { showing } \\
\text { increase after } \\
\text { Monsoon }(\%)\end{array}$ \\
\hline 1 & Total Dissolved salt & $778-4732$ & $647.3-216.9$ & 500 & 100 & 100 & Dec-57 & 29 \\
\hline 2 & Chloride as Cl & $59-2152$ & $156-827$ & 250 & 71 & 85 & $5-223$ & 57 \\
\hline 3 & Total Alkalinity & $126-410$ & $143-379$ & 200 & 79 & 71 & $2.8-200$ & 43 \\
\hline 4 & Total hardness as $\mathrm{CaCo}_{3}$ & $212-1218$ & $234-556$ & 300 & 71 & 71 & Oct-41 & 35 \\
\hline 5 & Calcium as $\mathrm{Ca}^{2+}$ & $134-376$ & $123-327$ & 75 & 100 & 100 & $10-23.5$ & 29 \\
\hline 6 & Magnesium as $\mathrm{Mg}^{2+}$ & $72-1063$ & $72-259$ & 30 & 100 & 100 & Jun-69 & 43 \\
\hline 7 & Silicates as $\mathrm{SiO}_{2}$ & $5.41-27.6$ & $0.09-27.05$ & & & & $8-102$ & 36 \\
\hline 8 & Sulphates as $\mathrm{So}_{4}{ }^{2-}$ & $5.05-25.24$ & $4.21-153.35$ & 200 & 7 & 0 & $30.5-48$ & 43 \\
\hline 9 & Nitrates as $\mathrm{NO}_{3}{ }^{-}$ & $2.93-9.84$ & $0.1-27.7$ & 45 & 0 & 0 & $4.8-320$ & 50 \\
\hline 10 & Sodium & $74.17-480$ & $190.9-754.5$ & 200 & $71 \%$ & 93 & $6-917$ & 50 \\
\hline 11 & Potassium & $2.74-120$ & $0-19.45$ & 1 & 100 & 100 & $35-162$ & 64 \\
\hline 12 & Fluoride as $\mathrm{F}^{-}$ & $0.219-1.35$ & $0.3-0.84$ & 1 & 7 & 0 & $7-105$ & 71 \\
\hline
\end{tabular}

\section{Heavy Metals}

\begin{tabular}{|c|c|c|c|c|c|c|c|c|}
\hline Sr.No. & $\begin{array}{c}\text { Parameters } \\
\text { (all values } \\
\text { one in mg/l })\end{array}$ & $\begin{array}{c}\text { Range Pre- } \\
\text { Mon }\end{array}$ & $\begin{array}{c}\text { Range Post- } \\
\text { Mon }\end{array}$ & Std BIS & $\begin{array}{c}\text { \% samples } \\
\text { exceeding } \\
\text { desired } \\
\text { limit- pre } \\
\text { monsoon }\end{array}$ & $\begin{array}{c}\text { \% samples } \\
\text { exceeding } \\
\text { desired } \\
\text { limit- post } \\
\text { monsoon }\end{array}$ & $\begin{array}{c}\text { Range of } \\
\text { Increase } \\
\text { Concentration } \\
\text { after monsoon } \\
(\%)\end{array}$ & $\begin{array}{c}\text { sample showing } \\
\text { increase after } \\
\text { Monsoon (\%) }\end{array}$ \\
\hline 1 & Iron & $0-7.27$ & $0.06-0.567$ & 0.3 & 29 & 36 & $35-210$ & 29 \\
\hline 2 & Nickel & $0.0005-0.036$ & $0-0.118$ & 0.02 & 7 & 71 & $31-50951$ & 71 \\
\hline 3 & Manganese & $0-0.323$ & $0.008-0.065$ & 0.1 & 0 & 0 & $100-258$ & 29 \\
\hline 4 & Cadmium & $0.0004-0.02$ & $0.002-0.015$ & 0.01 & 14 & 0 & $31-687$ & 50 \\
\hline 5 & Lead & $0-0.08$ & $0.019-0.06$ & 0.01 & 36 & 71 & $113-2143$ & 57 \\
\hline 6 & Copper & $0-0.172$ & $0.00-10.009$ & 0.05 & 14 & 36 & $8-308$ & 36 \\
\hline 7 & Zinc & $0.0075-0.224$ & $0-0.0 .22$ & 5 & 0 & 0 & $1000-1162$ & 43 \\
\hline
\end{tabular}

Surface Water( 7 Samples)

\begin{tabular}{|c|c|c|c|c|c|c|c|c|}
\hline & $\begin{array}{c}\text { Parameters( all values one } \\
\text { in mg/l ) }\end{array}$ & $\begin{array}{c}\text { Range Pre- } \\
\text { Mon }\end{array}$ & $\begin{array}{c}\text { Range Post- } \\
\text { Mon }\end{array}$ & Std BIS & $\begin{array}{c}\text { \% samples } \\
\text { exceeding } \\
\text { desired } \\
\text { limit- pre } \\
\text { monsoon }\end{array}$ & $\begin{array}{c}\text { \% samples } \\
\text { exceeding } \\
\text { desired } \\
\text { limit- post } \\
\text { monsoon }\end{array}$ & $\begin{array}{c}\text { Range of } \\
\text { Increase } \\
\text { Concentration } \\
\text { after } \\
\text { monsoon (\%) }\end{array}$ & $\begin{array}{c}\text { sample showing } \\
\text { increase after } \\
\text { Monsoon (\%) }\end{array}$ \\
\hline 1 & $\mathrm{pH}$ & $7.3-8.8$ & $8-9.32$ & $6.5-8.5$ & & & & \\
\hline 2 & Total Dissolved salt & $182.5-2368$ & 182 & 500 & 100 & 100 & 0 & 0 \\
\hline 3 & Chloride as Cl & $4-10740$ & $31-98$ & 250 & 57 & 42 & $0-675$ & 14 \\
\hline 4 & Total Alkalinity & $164-697$ & $139-271$ & 200 & 71 & 14 & & \\
\hline 5 & Total hardness as CaCo3 & $103-3644$ & $53-488$ & 300 & 57 & 14 & $0-14.2$ & 74 \\
\hline 6 & Calcium as $\mathrm{Ca}^{2+}$ & $43-175$ & $47-137$ & 75 & 71 & 28 & $9-111.8$ & 28 \\
\hline 7 & Magnesium as $\mathrm{Mg}^{2+}$ & $53-3469$ & $6-351$ & 30 & 100 & 85 & $0-14.2$ & 36 \\
\hline 8 & Silicates as $\mathrm{SiO}_{2}$ & $1.74-12.235$ & $0-50.67$ & 17 & & & $27-888$ & 57 \\
\hline 9 & Sulphates as $\mathrm{So}_{4}{ }^{2-}$ & $5.48-790.78$ & $2.94-46.343$ & 200 & 0 & 0 & 0 & 0 \\
\hline 10 & Nitrates as $\mathrm{NO}_{3}^{-}$ & $0-39.98$ & $0-2.52$ & 45 & 0 & 0 & $102-240$ & 28 \\
\hline 11 & Sodium & $2.98-897$ & $72.72-500$ & 200 & 57 & 57 & $19-48$ & 28 \\
\hline 12 & Potassium & $5.48-35$ & $3.69-60.86$ & 1 & 100 & 100 & $24-295$ & 28 \\
\hline 13 & Fluoride as $\mathrm{F}^{-}$ & $0.48-2.57$ & $0.28-1.43$ & 1 & 28 & 28 & $0.8-48$ & 28 \\
\hline
\end{tabular}

Volume 5 Issue 6, June 2016 www.ijsr.net

Licensed Under Creative Commons Attribution CC BY 


\section{International Journal of Science and Research (IJSR) \\ ISSN (Online): 2319-7064}

Index Copernicus Value (2013): 6.14 | Impact Factor (2015): 6.391

\begin{tabular}{|c|c|c|c|c|c|c|c|c|}
\hline Sr. No. & $\begin{array}{c}\text { Parameters( } \\
\text { all values one } \\
\text { in mg/l })\end{array}$ & $\begin{array}{c}\text { Range Pre- } \\
\text { Mon }\end{array}$ & $\begin{array}{c}\text { Range Post- } \\
\text { Mon }\end{array}$ & Std BIS & $\begin{array}{c}\% \text { samples } \\
\text { exceeding } \\
\text { desired limit- } \\
\text { pre monsoon }\end{array}$ & $\begin{array}{c}\% \text { samples } \\
\text { exceeding } \\
\text { desired limit- } \\
\text { post } \\
\text { monsoon }\end{array}$ & $\begin{array}{c}\text { Range of } \\
\text { Increase } \\
\text { Concentration } \\
\text { after monsoon } \\
(\%)\end{array}$ & $\begin{array}{c}\text { sample } \\
\text { showing } \\
\text { increase after } \\
\text { Monsoon } \\
(\%)\end{array}$ \\
\hline 1 & Iron & $0.054-0.13$ & $0.195-0.9$ & 0.3 & 71 & 42 & $22-1846$ & 57 \\
\hline 2 & Nickel & $0.0004-0.096$ & $0-0.118$ & 0.02 & 0 & 28 & $23-17668$ & 71 \\
\hline 3 & Manganese & $0-3.37$ & $0.015-0.08-$ & 0.1 & 28 & 0 & $0-40$ & 14 \\
\hline 4 & Cadmium & $0-0.027$ & $0.0017-0.0078$ & 0.01 & 28 & 0 & $0-57$ & 14 \\
\hline 5 & Lead & $0.003-0.11$ & $0-0.028$ & 0.01 & 14 & 0 & $60-220$ & 42 \\
\hline 6 & Copper & $0.004-0.38$ & $0.002-0.005$ & 0.05 & 28 & 0 & 0 & 0 \\
\hline 7 & Zinc & $0.054-0.022$ & $0.08-0.3$ & 5 & 0 & 0 & $52-460$ & 42 \\
\hline
\end{tabular}

Down pour in Gandhidham from $16^{\text {th }}$ January till $22^{\text {nd }}$ of September 2015 was $450 \mathrm{~mm}$, .Concentration of various Parameters has been found increased between April 2015 to November 2015. Following Graphical representation shows the dilution of different parameters. Negative values show increased concentration while Positive values shows dilution.

\section{Ground Water}
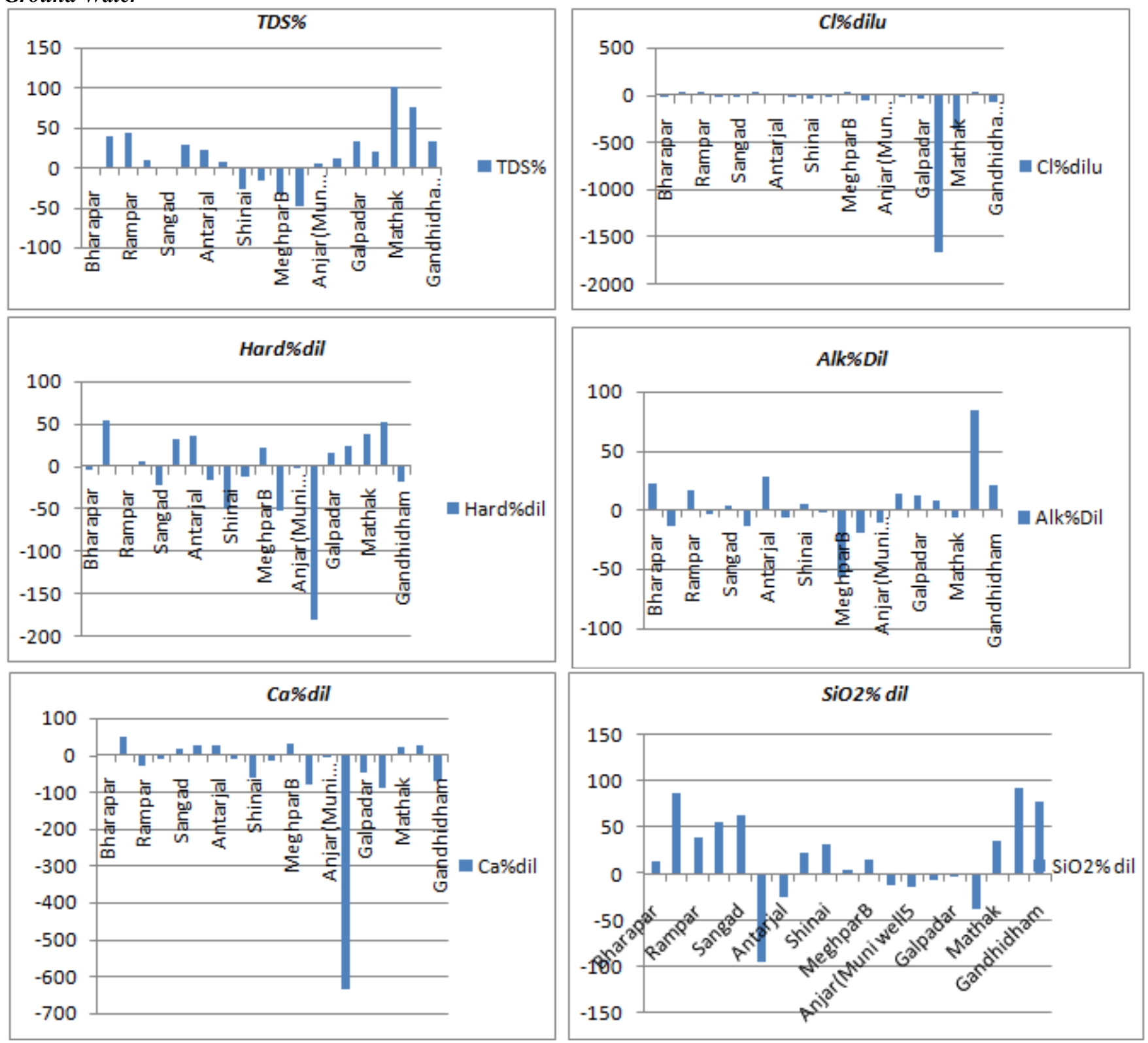

\section{Volume 5 Issue 6, June 2016 www.ijsr.net}

Licensed Under Creative Commons Attribution CC BY 
International Journal of Science and Research (IJSR)

ISSN (Online): 2319-7064

Index Copernicus Value (2013): 6.14 | Impact Factor (2015): 6.391
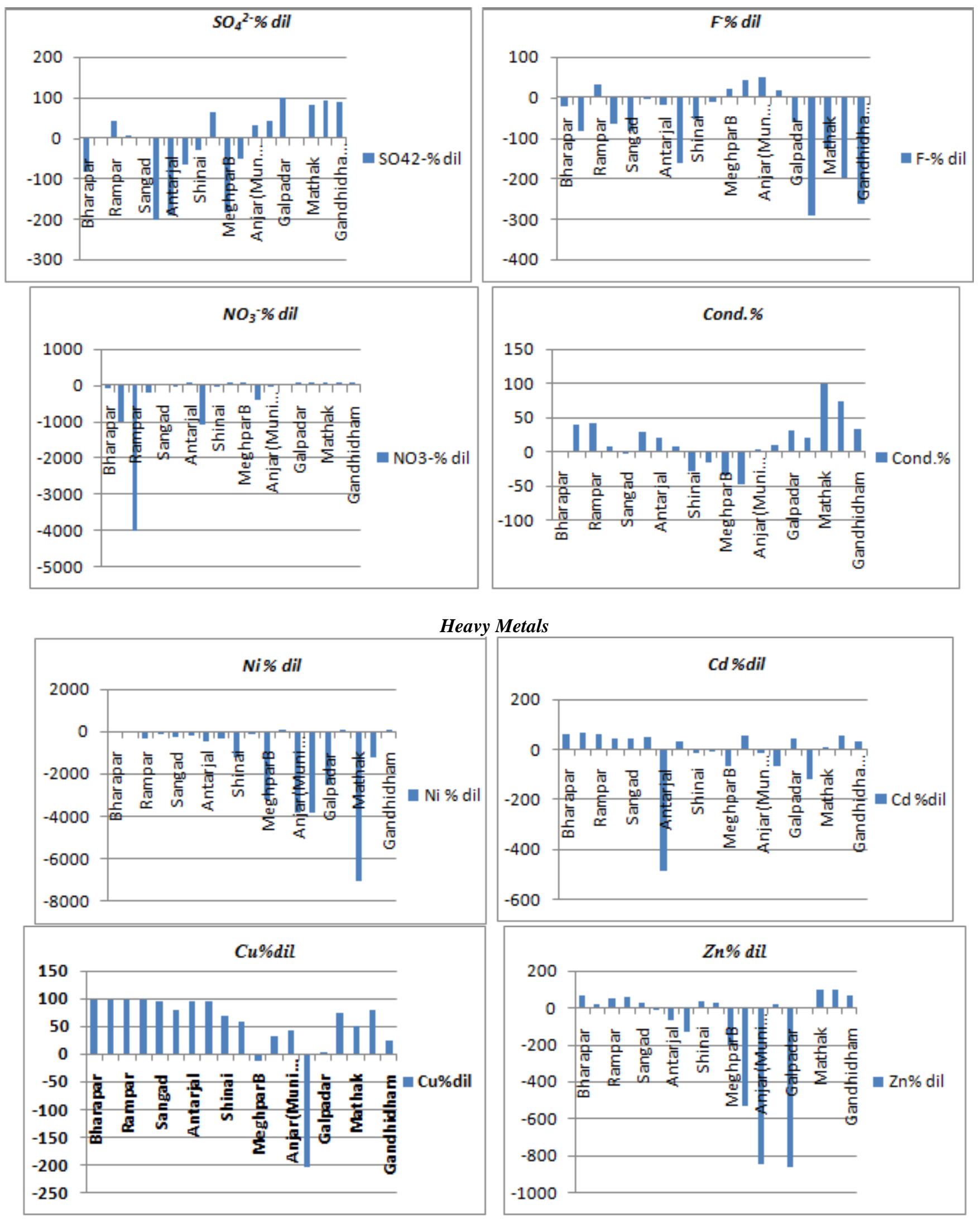

\section{Volume 5 Issue 6, June 2016} www.jisr.net

Licensed Under Creative Commons Attribution CC BY 


\section{International Journal of Science and Research (IJSR) \\ ISSN (Online): 2319-7064}

Index Copernicus Value (2013): 6.14 | Impact Factor (2015): 6.391
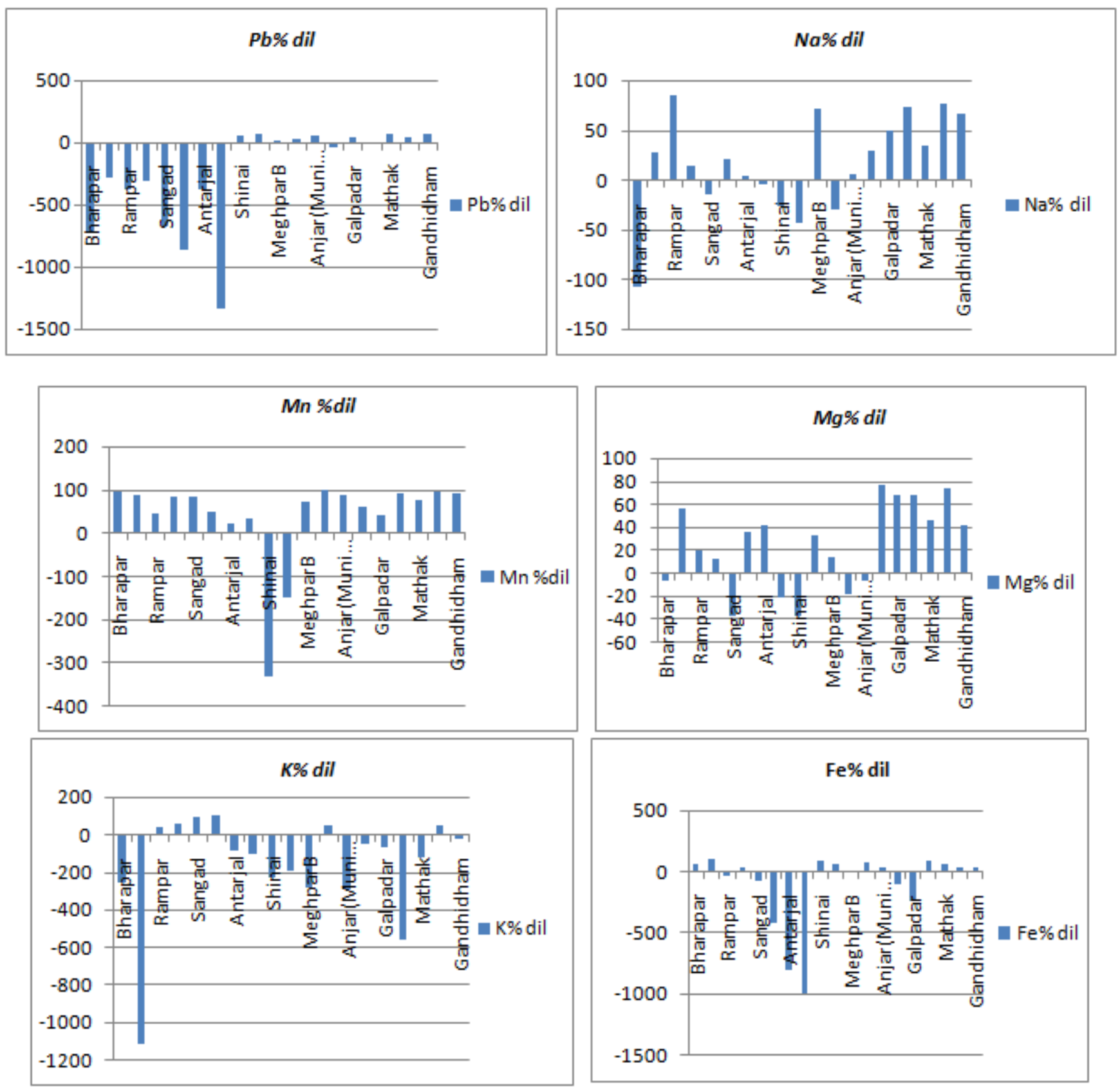

\section{Conclusion}

In comparative study of Ground Water it is found that $26 \%$ of samples showed increased TDS. Mostly Calcium, Potassium Nitrates chloride ions, and fluoride ions are washed off with rain water and leached to ground water table. $71 \%$ samples of ground water showed increased concentration of Fluoride after monsoon. 68\% samples have increased concentration of Potassium which is in the range of 21 to $1107 \%$ more than the pre- monsoon. $\mathrm{Cl}^{-}$in $68 \%$ samples and in $57 \%$ samples $\mathrm{No}_{3}$ is found to be increased after Monsoon.

Among heavy metals $\mathrm{Pb}$ and $\mathrm{Ni}$ showed maximum increase in the concentration in post Monsoon.

In Potable water which is mainly ground Water from Nangalpar, Viri and surface water from Tappar dam the concentration of fluoride (71\% Samples), K (64\% samples) , $\mathrm{Mg}(43 \%$ samples ) is found to be increased. $57 \%$ samples showed increase in $\mathrm{Pb}$ concentration and $71 \%$ samples showed increased Ni concentration.

After monsoon $71 \%$ of the samples showed more than desired limits are really alarming as this water is used for human consumption. In surface water $71 \%$ of the samples showed increase in concentration of nickel after Monsoon but under desired limit.

\section{Remedies}

1) More and more check dams should be constructed but only after checking the percolation rate of the soil layers and soil texture so as to increase the water percolation with less concentration of metallic and non-metallic constituents which makes water unfit for consumption.

2) Construction of the bore wells i.e. exploration of the ground water should be strictly under government authorities to control haphazard use of Ground water and

\section{Volume 5 Issue 6, June 2016 www.ijsr.net}




\section{International Journal of Science and Research (IJSR) \\ ISSN (Online): 2319-7064}

Index Copernicus Value (2013): 6.14 | Impact Factor (2015): 6.391

it must be made compulsory to have provisions for recharge of Ground water along with drilling bore well.

3) As it is found that the concentration of Lead and Nickel increased after the down pour concludes that $\mathrm{Pb}$ and Nickel has been washed along with water from the land sources which may be either industrial or garbage .Nickel and Lead are mainly used in Battery cells for domestic electric supports or in vehicles. Most of the areas showing increased concentration of these metals are residential and even if they are industrial, they have small scale industries which is also very less in Number. Municipal authorities should take initiative for proper disposal of E Garbage.

4) Area around the dams should be cleaned especially before monsoon so that washing off of pollutants from waste can be avoided

\section{Acknowledgement}

The authors would like to thank IFFCO, Kandla, Kachchh, , Gujarat for providing necessary Laboratory facilities and for the support to conduct this work.

\section{References}

[1] Abolude David Sunday , Davies OnomeAugustina, Barak Zebedee , OpabunmiOlatunbosunOlajideAnalyses of Heavy Metals in Water and Sediment of Bindare Stream, Chikaji Industrial Area SabonGari

[2] Central Pollution Control Board (Ministry of Environment and forests)- Status of ground water quality in India- Part-1- a Report (2006-2007)

[3] D. M. Costa \& L. F. Melo\& F. G. Martins-Localization of Contamination Sources in Drinking Water Distribution Systems: A Method Based on Successive Positive Readings of Sensors

[4] Hazardous metals and minerals Pollution in India: Sources, toxicity

[5] M. Dinesh Kumar and Tushaar Shah- Groundwater Pollution and Contamination in India:.

[6] N.C. Kankal, M.M. Indurkar, S.K. Gudadhe and S.R. Wate-Water QualitySuresh Nagar - Groundwater and well-water quality in Alluvial aquifer of Central Gujarat

[7] Sunderrajan Krishnan1, Sanjiv Kumar, DoekeKampman and Suresh Nagar-Groundwater and well-water quality in Alluvial aquifer of Central Gujarat.

[8] Index of Surface Water Bodies of Gujarat, India

[9] Sajid Farid1, Musa KaleemBaloch and Syed Amjad Ahmad-Water pollution: Major issue in urban areas

\section{Author Profile}

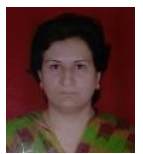

Ms Madhu D Nathani is Research Scholar, Department of Earth and Environment, KSKV. Kachchh University Bhuj, Kachchh, Gujarat, India

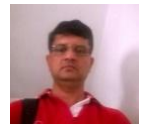

Dr. Mrugesh H. Trivedi is Toxicologist, Worked on impact of fluoride toxicity on mice brain and its possible alleviation by black tea extract. Extrapolated that work on humans. He is Assistant Professor, Department of Earth and Environment KSKV. Kachchh University. Bhuj. Kachchh . Gujarat, India

Volume 5 Issue 6, June 2016 www.ijsr.net 